Meta

Journal des traducteurs

Translators' Journal

\title{
Traduction et politique canadienne : quel est le rôle des journalistes entre 1942 et 1995 ?
}

\section{Chantal Gagnon}

Volume 58, numéro 3, décembre 2013

URI : https://id.erudit.org/iderudit/1025049ar

DOI : https://doi.org/10.7202/1025049ar

Aller au sommaire du numéro

\section{Éditeur(s)}

Les Presses de l’Université de Montréal

ISSN

0026-0452 (imprimé)

1492-1421 (numérique)

Découvrir la revue

Citer cet article

Gagnon, C. (2013). Traduction et politique canadienne : quel est le rôle des journalistes entre 1942 et 1995 ? Meta, 58(3), 522-541.

https://doi.org/10.7202/1025049ar

\section{Résumé de l'article}

La présente étude tente de jeter un éclairage nouveau sur l'apport des journalistes à la question du bilinguisme. Plus précisément, l'article porte sur la traduction politique dans les médias écrits canadiens. À certains égards, les journalistes restructurent la relation entre le public et le gouvernement en mettant au jour certains des mécanismes qui régissent les processus gouvernementaux de communication. Cet état de fait montre notamment que les phénomènes du bilinguisme officiel et de la traduction institutionnelle ne sont pas nécessairement dissimulés à la collectivité, puisque les grands quotidiens y prêtent attention en situation de crise. Le corpus de travail rassemble des articles rédigés entre 1942 et 1995, à propos de discours politiques traduits. La traduction de ces discours a été commentée d'une façon ou d'une autre dans les médias écrits au Québec et au Canada. Les quotidiens suivants ont été consultés : La Presse, Le Devoir, The Gazette et The Globe and Mail. L'étude montre que les journalistes canadiens et québécois sont conscients des écarts entre les versions linguistiques des discours de leurs dirigeants. Trois rôles sont identifiés chez le journaliste : le journaliste-témoin du phénomène de la traduction, le journaliste-commentateur de la traduction et le journaliste-traducteur de discours politique.
Tous droits réservés @ Les Presses de l’Université de Montréal, 2014
Ce document est protégé par la loi sur le droit d'auteur. L’utilisation des services d'Érudit (y compris la reproduction) est assujettie à sa politique d'utilisation que vous pouvez consulter en ligne.

https://apropos.erudit.org/fr/usagers/politique-dutilisation/ 


\title{
Traduction et politique canadienne: quel est le rôle des journalistes entre 1942 et 1995 ?
}

\author{
CHANTAL GAGNON \\ Université de Montréal, Montréal, Canada \\ chantal.gagnon.4@umontreal.ca
}

\begin{abstract}
RÉSUMÉ
La présente étude tente de jeter un éclairage nouveau sur l'apport des journalistes à la question du bilinguisme. Plus précisément, l'article porte sur la traduction politique dans les médias écrits canadiens. À certains égards, les journalistes restructurent la relation entre le public et le gouvernement en mettant au jour certains des mécanismes qui régissent les processus gouvernementaux de communication. Cet état de fait montre notamment que les phénomènes du bilinguisme officiel et de la traduction institutionnelle ne sont pas nécessairement dissimulés à la collectivité, puisque les grands quotidiens y prêtent attention en situation de crise. Le corpus de travail rassemble des articles rédigés entre 1942 et 1995, à propos de discours politiques traduits. La traduction de ces discours a été commentée d'une façon ou d'une autre dans les médias écrits au Québec et au Canada. Les quotidiens suivants ont été consultés: La Presse, Le Devoir, The Gazette et The Globe and Mail. L'étude montre que les journalistes canadiens et québécois sont conscients des écarts entre les versions linguistiques des discours de leurs dirigeants. Trois rôles sont identifiés chez le journaliste: le journaliste-témoin du phénomène de la traduction, le journaliste-commentateur de la traduction et le journaliste-traducteur de discours politique.
\end{abstract}

\section{ABSTRACT}

This study will attempt to shed new light on the contribution of journalists to the issue of bilingualism. More specifically, the article will focus on the translation policy in Canadian print media. In some respects, journalists restructure the relationship between the public and the government in uncovering some of the mechanisms that govern the process of government communication. This shows in particular that the phenomena of bilingualism and institutional translation are not necessarily hidden from the community, since major newspapers pay attention to them in crisis situation. The research uses a corpus of articles written between 1942 and 1995, each article discussing issues about a translated political speech. It has been found that in one way or another, the translation of political speeches has been commented upon in the print media in Quebec and Canada. The following daily newspapers were used in the study: La Presse, Le Devoir, The Gazette and The Globe and Mail. It would appear that journalists in Quebec and Canada are aware of the differences between the language versions of their leaders' speeches. Three roles are identified for journalists dealing with translation: the journalist-witness of the translation phenomenon, the journalist-commentator of the translation and the journalist-translator of political discourse.

\section{MOTS-CLÉS/KEYWORDS}

discours politique, traduction, médias, journalistes, réception political speeches, translation, media, journalists, reception 


\section{Introduction}

Le rôle des médias dans une société démocratique est inévitablement associé à l'information et à la critique. Selon Bernier, le journaliste est un «acteur social» qui doit assumer certaines responsabilités afin de servir l'intérêt public, ce qui l'amène à «chercher et diffuser de façon équitable et rigoureuse des informations véridiques et pertinentes qui aideront l'ensemble des citoyens d'une société à faire [...] des choix éclairés visant à améliorer les conditions de vie» (Bernier 2004: 8).

Cependant, puisque les médias influencent les attitudes et les opinions des sociétés, ils participent aussi à une certaine construction de la réalité. Les médias n'échappent pas non plus à la propagande et à la collusion avec l'oppression militarocapitaliste (Herman et Chomsky 1998). Nombre de chercheurs estiment que les médias reproduisent les idéologies des groupes dominants (par exemple, Fairclough 1995 ou Van Dijk 1988). Les journaux canadiens et québécois ne sont pas exempts de ce phénomène: comme l'ont remarqué les chercheurs Potvin (1999), Robinson (1998) et Conway (2011), c'est particulièrement frappant dans le cas des conflits qui opposent les deux communautés linguistiques officielles du Canada. Ainsi, Potvin estime qu'à la suite du référendum de 1995, il existait au Canada anglais un discours aux tendances racistes envers le Québec. Robinson, elle, affirme que la couverture médiatique du référendum de 1980 ne s'est pas déroulée sans parti pris, tant chez les francophones que chez les anglophones. Quant aux travaux de Kyle Conway (2011), ils ont bien montré qu'en tant que télédiffuseurs publics, Radio-Canada et CBC ont pour mission d'aider les deux solitudes canadiennes à s'apprivoiser l'une l'autre, mais que cette mission est entravée par des obstacles historiquement conditionnés. Pour Conway, le phénomène de la traduction culturelle figure au cœur de la problématique.

En projetant une certaine image de l'autre communauté linguistique au Canada, les journaux influent sur la relation conflictuelle entre les francophones et les anglophones du pays. L'extrait suivant illustre bien à quel point la question linguistique alimente les tensions entre les communautés, et ce, même entre journalistes:

Mes collègues anglophones d'Ottawa [...] éteignent systématiquement leur enregistreuse quand on passe en français dans les points de presse. Vous posez une question en français et vous entendez soudainement une douzaine d'appareils faire clac! clac! clac!

Vous direz peut-être que je suis susceptible, mais je prends toujours ces clacs! comme autant de claques sur ma gueule de francophone. Le pire, c'est que les mêmes collègues viennent nous demander par la suite s'ils ont manqué quelque chose d'important. $\left(\right.$ Marissal 2002: B2) ${ }^{1}$

Le chroniqueur politique Vincent Marissal, un habitué de la «colline parlementaire» canadienne, évoque ici les difficiles relations entre francophones et anglophones. D'un point de vue traductologique, il y a lieu de se demander si ces difficultés ont des répercussions sur la réception des discours politiques traduits dans les médias canadiens. Plus précisément, on peut s'interroger sur le rôle occupé par les journalistes dans le domaine de la traduction des discours politiques. En effet, l'activité de traduction est au cœur de la politique canadienne, puisque les discours du Premier ministre fédéral et ceux de ses ministres sont généralement traduits, ainsi que tous les discours parlementaires de la Chambre des communes. La fréquence de l'activité de traduction en politique canadienne a sans doute des incidences sur la façon dont les journalistes traitent des discours dans leurs écrits. 
Nos recherches précédentes ont établi qu'au Canada, on retrouve à l'occasion des écarts de traduction entre les allocutions française et anglaise des dirigeants du pays (Gagnon 2006a; 2006b). Grâce à ces écarts, un gouvernement a la possibilité de modifier légèrement son message en fonction de la communauté linguistique à laquelle il s'adresse. Il importe donc de vérifier comment, dans leurs écrits, les journalistes abordent la traduction des allocutions des élus, puisque l'un des objectifs du journalisme est de présenter un portrait juste d'une réalité donnée, manœuvres politiques incluses.

Dans un article précédent, nous avons exploré le thème de la visibilité de la traduction dans la presse politique canadienne (Gagnon 2012). À bien des égards, la présente étude constitue la suite de cet article, puisque nous traitons aussi de la traduction dans la presse politique canadienne. Cette fois, nous proposons deux pistes de réflexion, présentées ici sous forme de questions:

1) Puisqu'il semble exister un mur entre les deux communautés linguistiques au Canada, les journalistes de ces communautés tiennent-ils compte des différences entre les versions française et anglaise des discours du Premier ministre ou s'en tiennent-ils à la version rédigée dans leur langue maternelle?

2) Lorsqu'il y a absence de traduction, comment les journalistes interviennent-ils dans les textes politiques qu'ils citent (traduction, paraphrase interlinguistique...)?

Nous aborderons chacune de ces questions dans une section séparée (section 2 et 3). Il s'agira non seulement de mieux comprendre la façon dont les journalistes commentent les discours politiques traduits en contexte canadien, mais aussi d'évaluer les stratégies utilisées lorsqu'ils traduisent eux-mêmes des extraits de discours.

Le corpus à l'étude sera le même que dans Gagnon (2012), soit des articles de journaux rédigés à la suite de discours politiques importants, en situation de crise nationale au Canada (le plébiscite canadien sur la conscription outre-mer en 1942, la Crise d'octobre en 1970, l'arrivée au pouvoir d'un parti indépendantiste au gouvernement du Québec en 1976, le premier référendum sur la souveraineté du Québec en 1980, l'échec le l'Accord du Lac Meech en 1990 et finalement, le second référendum sur la souveraineté du Québec en 1995). Les articles étudiés sont présentés à l'Annexe 1. Ces articles proviennent de quatre grands quotidiens canadiens, deux d'expression française (Le Devoir et La Presse) et deux d'expression anglaise (The Gazette et The Globe and Mail).

\section{Traductions commentées}

Pour rédiger leur compte rendu d'une allocution politique, les journalistes ont généralement recours aux communiqués du gouvernement fédéral. Or, ces communiqués ne sont généralement pas bilingues (Gagnon 2009; 2012): les versions française et anglaise d'un même discours sont présentés aux médias sous la forme de deux communiqués distincts, l'un en français et l'autre en anglais. Cette pratique a pour effet (sciemment ou non) de décourager la comparaison entre les versions linguistiques des discours. D’ailleurs, dans la première partie de notre étude (Gagnon 2012), nous avons vu que sur les 106 articles recensés, seuls 6 articles présentaient un commentaire sur les traductions des discours.

Il existe de nombreuses recherches sur l'utilisation que font les journalistes des communiqués de presse. La plupart des chercheurs (par exemple: Jacobs 1999; Davies 
2008, Pander Maat 2007; Herman 2009; Van Hout, Pander Maat et De Preter 2011) établissent le caractère propagandiste ou autopromotionnel du communiqué de presse. Bien des auteurs évoquent aussi le fait qu'il existe une grande intertextualité entre les communiqués de presse et les articles rédigés à partir de ceux-ci. C’est donc que les communiqués sont très utilisés, parfois trop comme le note le journaliste d'expérience Nick Davies. Pour ce dernier, les médias écrits sont malades, puisque les journalistes recyclent les communiqués de presse sans remettre en question ce qui y est écrit (Davies 2008: 59). D’autres chercheurs prennent leurs distances des critiques de Davies: selon eux, les journalistes réussissent à maintenir un point de vue critique dans leurs écrits, même si une partie de leurs renseignements proviennent des communiqués (voir Herman 2009; Van Hout, Pander Maat et De Preter 2011).

Dans son livre Preformulating the News, Jacobs explique que les caractéristiques structurelles du communiqué de presse reflètent la visée de ce type de texte, à savoir inciter les médias à retranscrire tel quel un message bien précis (Jacobs 1999: 20). Ainsi, le titre «communiqué de presse» inscrit au début du document et l'expression «fin du communiqué de presse» au bas de la feuille délimitent la portion destinée à être recopiée par le journaliste. Comme le précise Jacobs (1999: 20-21) : «In particular, [these regular features] serve to signal to the journalists that what follows or precedes is not really meant for them, but that it is supposed to be copied.»

C'est donc que la structure même du communiqué incite les journalistes à retranscrire un message donné sans modifier son contenu. Sachant que les communiqués unilingues du gouvernement fédéral découragent la comparaison entre les versions linguistiques des discours, on peut se demander si les journalistes canadiens acceptent telle quelle la traduction du gouvernement fédéral. Les journalistes arriventils à maintenir un point de vue critique par rapport à la traduction de l'institution fédérale?

En 1970, le journaliste Claude Turcotte de La Presse explique à son lectorat que les versions française et anglaise du discours de Trudeau étaient identiques «à quelques détails près» (Turcotte 1970: A1, voir annexe). Il est évidemment difficile de définir ce qu'entend Turcotte en utilisant l'expression «détails», mais on peut s'entendre sur le fait que selon lui, il s'agissait de divergences mineures. Voyons quelques exemples des écarts entre les versions anglaise et française du discours en question (dans cet article, tous les soulignements sont de nous):

(1) a. The governments of Canada and Quebec have been told by groups of self-styled revolutionaries that they intend to murder in cold blood two innocent men unless their demands are met.

(Canada, Prime minister 1970a: 1; nous soulignons) ${ }^{2}$

b. Les gouvernements du Canada et du Québec se sont fait dire par des groupes de révolutionnaires que, si l'on ne donne pas suite à leurs demandes, deux hommes seront assassinés.

(Canada, Premier ministre 1970b: 2) ${ }^{3}$

(2) a. Who are these men who are held out as latter-day patriots and martyrs?

(Canada, Prime minister 1970a: 2; voir note 2; nous soulignons)

b. Qui sont-ils, ces individus qu'on voudrait nous faire passer pour des patriotes et des martyrs?

(Canada, Premier ministre 1970b : 3; voir note 3) 
Dans la version anglaise des extraits (1) et (2), le Premier ministre Pierre Elliott Trudeau brosse un portrait à la fois dur et cynique des terroristes, chose qu'il ne fait pas en français. Autre fait important, 14 phrases du texte français ont été omises de la version anglaise dans le discours de Trudeau en 1970. Une telle omission n'est pas anodine puisqu'elle est relativement rare dans la traduction des discours politiques. D'ordinaire, la grande majorité des phrases d'un discours sont traduites. Ainsi, l'omission complète de 14 phrases ne peut être qualifiée de «détail».

En 1976, la traduction d'un autre message à la nation de Pierre Elliott Trudeau fut largement commentée par la journaliste Lise Bissonnette. Voici un extrait de son article, dans le journal Le Devoir:

(3) Si ce genre de discussion a cessé, c'est selon lui parce que «toutes les provinces» (certaines provinces, dans la version anglaise) ont préféré s'occuper plutôt du rapatriement et de la formule d'amendement de la constitution. [...]

Entre la version anglaise et la version française du texte de M. Trudeau, il y a ici et là, de petites et subtiles différences. Ne serait-ce que le "prix du pétrole», qui, dans le texte français, est soudainement qualifié de «beaucoup inférieur au prix mondial», par exemple. A l'inverse, le texte anglais fait plus souvent référence aux autres provinces, quant [sic] il est question de discussions entre partenaires.

Et parce qu'il y a effectivement de petites différences, le texte anglais de M. Trudeau a été disponible dès sept heures hier soir à quelques media anglophones, tandis que le texte français est arrivé aux media francophones pendant que le chef du gouvernement s'adressait à la nation, avec le privilège de l'en-tête officiel, faut-il dire.

(Bissonnette 1976: 1-6, voir annexe)

Ici, Lise Bissonnette indique au lecteur que le discours de la veille n'était pas identique dans les deux langues. Par ailleurs, elle illustre son propos d'exemples, ce qui est fort rare dans notre corpus. Plus précisément, il s'agit du seul cas où une journaliste analyse avec autant de minutie les différences entre les versions linguistiques d'une allocution politique. Le fait que le Cabinet du Premier ministre ait retardé la diffusion du communiqué français a sans doute incité la journaliste à comparer les versions du discours.

En 1980, deux journalistes évoquent eux aussi le phénomène de la traduction dans leur article: il s'agit de Claude Turcotte du Devoir et de Louise Cousineau de La Presse:

(4) Au début du message qu'il a lu fidèlement, en français d'abord, puis en anglais ensuite, M. Trudeau a remercié au nom de tous les Canadiens les fédéralistes qui ont travaillé à cette victoire et il a félicité très particulièrement $\mathrm{M}$. Claude Ryan [...].

(Turcotte 1980: 1, voir annexe)

(5) Les discours des chefs nous furent retransmis avec fidélité. TVA a toutefois commis une erreur en supprimant les parties anglaises de ceux de MM. Ryan et Trudeau, parce qu'on n'avait pas engagé d'interprètes pour les traduire. Parfois, les discours varient sensiblement selon l'auditoire auquel on s'adresse (ce n'était pas le cas hier soir) et un diffuseur sérieux en information ne doit pas supprimer un exercice agaçant (les traductions sont toujours ennuyeuses).

(Cousineau 1980: 11, voir annexe)

Seule Cousineau discute brièvement de la fidélité de la traduction, en précisant que les discours en anglais et en français ne présentaient pas de variations notables. Or, les extraits suivants tendent à prouver le contraire: 
(6) a. A majority of them have decided to reject sovereignty-association, and to express their loyalty to Canada.

(Canada, Prime minister 1980a: 1; nous soulignons ${ }^{4}$ )

b. Et les Québécois ont choisi majoritairement de rejeter la souveraineté-association et d'opter pour la voie de la fidélité au Canada.

(Canada, Premier ministre 1980b: 1; nous soulignons ${ }^{5}$ )

(7) a. That is why I am happy and relieved that the people of Quebec have put an end to doubt, and have proclaimed today by majority vote their devotion to Canada.

(Canada, Premier ministre 1980a: 2; nous soulignons; voir note 4)

b. Voilà pourquoi je suis heureux et soulagé que les Québécois et les Québécoises aient mis fin à leurs doutes et proclamé aujourd'hui, par un vote majoritaire, leur attachement au Canada.

(Canada, Premier ministre 1980b: 1; nous soulignons; voir note 5)

(8) a. However, I cannot put out of my mind all those «Yes» supporters who fought with such strong convictions, and who tonight have seen their option defeated by the verdict of the majority.

(Canada, Prime minister 1980a: 1; nous soulignons; voir note 4)

b. Pourtant je ne peux m'empêcher de penser à tous ces tenants du OUI qui se sont battus avec tant de conviction et qui doivent ce soir remballer leur rêve et se plier au verdict de la majorité.

(Canada, Premier ministre 1980b: 1; nous soulignons; voir note 5)

Dans les exemples ci-dessus, on constate un léger glissement entre les versions anglaise et française. Plus précisément, dans les exemples (6) et (7), la fidélité et l'attachement des Canadiens semblent plus grands en anglais qu'en français. Quant à l'exemple (8), il présente la défaite du mouvement souverainiste québécois avec un peu plus de compassion en français qu'en anglais. On peut penser que Louise Cousineau n'a pas vu ces écarts ou qu'elle a jugé qu'ils n'étaient pas assez importants pour être dignes de mention. Il est difficile de déterminer laquelle de ces hypothèses est la plus vraisemblable puisque la journaliste ne donne pas d'exemples pour illustrer son propos.

En 1990, aucun journaliste n'a commenté la traduction des discours de Brian Mulroney lors de l'échec de l'accord du lac Meech. Il faut dire que les écarts entre les versions françaises et anglaises des discours de Brian Mulroney étaient peut-être moins remarquables que ceux retrouvés dans les discours de Pierre Elliott Trudeau. Ainsi, seules deux phrases ont été omises de la traduction française, contrairement aux 14 phrases omises dans le discours de 1970. Cependant, les extraits suivants montrent bien qu'il existait des écarts d'importance entre les versions française et anglaise de Brian Mulroney:

(9) a. But Quebec emerged from these negotiations with its dignity and its principles intact.

(Canada, Prime minister 1990a: 2; nous soulignons ${ }^{6}$ )

b. Mais il [Québec] sort de ces négociations en ayant gardé sa dignité intacte et sans avoir le moindrement dérogé à ses principes.

(Canada, Premier ministre 1990b: 2; nous soulignons ${ }^{7}$ ) 
Dans l'exemple (9), le français met davantage l'accent que l'anglais sur le fait que le peuple québécois n’a pas dérogé à ses principes. Le rapatriement de la constitution en 1981 ayant été imposé au Québec par le gouvernement de Pierre Elliott Trudeau, il importait au Premier ministre Brian Mulroney de réparer ce qu'il considérait comme une erreur. Dans la version française, la question du respect des «principes» du Québec correspond à cette préoccupation de Brian Mulroney.

Lors de la campagne référendaire de 1995, les quotidiens La Presse, The Globe and Mail et The Gazette ont commenté dans leurs pages la traduction des allocutions de Jean Chrétien et de Lucien Bouchard, deux opposants ayant prononcé un discours le même jour. L'extrait suivant, tiré d'un article d'André Picard du Globe and Mail, est représentatif de cette tendance:

(10) Mr. Chrétien recorded his message in French and English in the Prime Minister's Office in Ottawa. [...] But the key to his speech, particularly in French, was a direct plea to undecided voters who, given that recent polls put the referendum opponents in a dead heat, probably will tip the balance.

(Picard 1995a: A1-A8, voir annexe)

Non seulement le journaliste fait-il mention des langues d'allocution du discours de Jean Chrétien, mais il précise qu'il existait des divergences entre les deux versions du discours. Soulignons que le discours de Jean Chrétien n'a pas attiré autant l'attention que celui de Lucien Bouchard, comme en font foi les deux extraits ci-dessous:

(11) Mais M. Bouchard avait un tout autre message, beaucoup moins incisif pour son auditoire anglophone. Alors que le Premier ministre a livré la même allocution en français et en anglais, le chef du Bloc québécois a réservé ses flèches contre Jean Chrétien pour sa présentation en français. En anglais, il a plutôt vanté les vertus du projet de partenariat Québec-Canada qu'il entend négocier advenant un OUI.

(Hébert 1995: A2, voir annexe)

(12) Bouchard speaks only in French of panic in Ottawa.

(Wells 1995: A11, voir annexe)

Si la presse écrite a autant commenté les discours de Lucien Bouchard, c'est sans doute parce qu'il existe des écarts flagrants entre les versions anglaise et française: notamment, la version française était bien plus longue que la version anglaise. Au gouvernement fédéral (dont Lucien Bouchard et Jean Chrétien faisaient partie), les hommes politiques présentent généralement des traductions similaires, du moins en apparence.

Les journalistes André Picard (The Globe and Mail) et Chantal Hébert (La Presse) arrivent à un constat différent en analysant la traduction du discours de Jean Chrétien (respectivement les exemples (10) et (11), ci-dessus). Picard remarque quelques différences, alors qu'Hébert affirme que les deux versions sont identiques. Afin de tirer cette situation au clair, étudions deux extraits du discours de Jean Chrétien en français et en anglais:

(13) a. Anyone who really wants to remain a Canadian should think twice before taking such a dangerous risk.

(Canada, Prime minister 1995a: 1; nous soulignons ${ }^{8}$ )

b. Ils ne devraient pas prendre un risque pareil.

(Canada, Premier ministre 1995b: 1; nous soulignons ${ }^{9}$ ) 
(14) a. When my fellow Quebecers make their choice on Monday, they have the responsibility and the duty to understand the implications of that choice. [...] Where Quebecers would no longer enjoy the rights and privileges associated with Canadian citizenship.

(Canada, Prime minister 1995a: 1; nous soulignons; voir note 8)

b. Quand nous ferons notre choix, nous avons tous la responsabilité et le devoir de comprendre la portée de notre décision. [...]

Nous, Québécois, ne serions plus Canadiens et n’aurions plus droit aux privilèges attachés à la citoyenneté canadienne [...].

(Canada, Premier ministre 1995b: 1; nous soulignons; voir note 9)

Dans l'exemple (13), le français est bien plus concis que l'anglais, au point où les deux versions envoient des messages différents. En effet, l'anglais explique qu'un vote pour un «OUI» constitue un risque sérieux pour qui veut rester Canadien. Le français tait ces arguments. Dans l'exemple (14), on voit que le point de vue utilisé par le Premier ministre n'est pas le même dans les deux versions linguistiques. En français, le Premier ministre s'adresse manifestement à ses compatriotes du Québec, alors qu'en anglais, il parle du peuple québécois à la troisième personne. Jusqu'à un certain point, une telle stratégie a pour effet d'exclure les minorités linguistiques du discours, particulièrement les Canadiens francophones à l'extérieur du Québec (voir aussi Gagnon 2006b). En effet, les Canadiens d'expression française à l'extérieur du Québec ne pouvaient pas voter et le message du Premier ministre ne s'adressait donc pas directement à cette minorité. Les exemples présentés ici nous amènent à conclure que les versions anglaise et française du discours de Jean Chrétien n'étaient pas identiques.

\subsection{Conclusion provisoire}

Dans notre corpus, on trouve à deux reprises un commentaire substantiel sur les traductions du gouvernement fédéral. Dans les deux cas, des indicateurs avaient mené les journalistes sur cette voie: le retard de la version française en 1976 et le discours nettement plus long en français du chef de l'Opposition en 1995. Même si des composantes incitent les journalistes à analyser les traductions gouvernementales, il reste que ces analyses existent. Ainsi, comme nous l'avions établi dans notre article précédent, non seulement les journalistes sont-ils intéressés par le phénomène de la traduction (voir Gagnon 2012), mais un petit nombre de journalistes vont jusqu'à commenter les textes traduits. En conséquence, les journalistes ne recyclent pas toujours aveuglément les communiqués de presse. Nos recherches semblent donc aller dans le sens de celles de Herman (2009) et de Van Hout, Pander Maat et De Preter (2011), à savoir que les journalistes expriment à l'occasion un point de vue critique par rapport aux communiqués de presse. Cependant, les commentaires des journalistes sur la traduction manquent parfois de justesse ou de précision. Dans la réalité, les traductions fédérales sont rarement remises en question avec efficacité.

Précisons qu'en 1995, dans le discours de Lucien Bouchard, les divergences entre les versions française et anglaise étaient telles que The Globe and Mail a traduit en anglais la version française du texte de Lucien Bouchard: 
(15) The following is an abridged translation of the French version of Bloc Québécois Leader Lucien Bouchard's speech last night.

(The Globe and Mail 1995a: A16, voir annexe)

Il s'agit ici d'une traduction de journaliste. Ainsi, les journalistes ne se contentent pas de commenter la traduction, ils participent également au processus. La prochaine section portera justement sur cette question.

\section{Interventions traductionnelles}

Dans un pays comme le Canada, où les différents leaders politiques s'expriment quotidiennement dans les deux langues officielles, les journalistes sont souvent aux prises avec la question du bilinguisme canadien: comment en effet rapporter les paroles d'un homme politique qui ne s'est pas exprimé dans la langue du lectorat? On a vu à la section précédente qu'il arrive aux journalistes de commenter les traductions fournies par l'institution gouvernementale, mais que faire lorsque le texte n'est pas traduit? Il existe en effet une foule d'occasions où les discours politiques ne sont pas traduits, même au Canada. C'est le cas, notamment, d'une majorité des discours des premiers ministres de province. C'est généralement le cas aussi des entrevues accordées par les dirigeants politiques du gouvernement fédéral ou provincial.

Dans notre corpus, les articles de journaux qui traitent de discours non traduits ciblent les 5 événements suivants:

- l'entrevue accordée par Pierre Elliott Trudeau à deux journalistes d'expression anglaise le 14 octobre 1970 ;

- le discours du Premier ministre du Québec René Lévesque au soir du référendum de 1980;

- le discours d'un autre Premier ministre du Québec, Robert Bourassa, à la suite de l'échec de l'accord du lac Meech;

- le discours du chef de l'Opposition de la Chambre des communes, Lucien Bouchard, peu de temps avant le référendum en 1995;

- le discours du Premier ministre du Québec, Jacques Parizeau, au soir du référendum de 1995.

Ces articles forment un sous-corpus de 12 articles, qui sous-tendent l'analyse dont fait état la présente section.

Nous qualifions d'intervention traductionnelle toute situation où un journaliste cite directement ou indirectement un discours politique prononcé en langue étrangère. Cinq types d'intervention traductionnelle sont proposés, soit la traduction, la nontraduction, la paraphrase interlinguistique, la précision en langue source et la citation bilingue. Avant de procéder à l'analyse de ces interventions, nous allons les définir.

La traduction correspond au transfert linguistique d'une citation directe, d'une langue source à une langue cible. Voici un exemple de traduction effectuée par Marcel Desjardins, un journaliste de La Presse en 1970. Ce journaliste de La Presse a traduit en français des extraits d'une entrevue de Pierre Elliott Trudeau:

(16) Le Premier ministre du Canada [...] est intervenu, mardi, pour affirmer que [...] «la société doit prendre tous les moyens à sa disposition pour se défendre contre l'émergence d'un pouvoir parallèle qui défie l'autorité élue du pays ».

(Desjardins 1970: F1, voir annexe) 
Par non-traduction, nous entendons une citation directe d'un discours, laissée en langue étrangère dans un article. L'extrait suivant, tiré de la même source que l'exemple précédent, illustre ce qu'est une non-traduction:

(17) Et pour M. Pierre Elliott Trudeau, ce recours peut être illimité. "To any distance», a-t-il répondu aux journalistes du réseau anglais de Radio-Canada qui l'interrogeaient sur la nécessité de recourir aux forces armées [...].

(Desjardins 1970: F1, voir annexe)

La non-traduction n'est pas phénomène unique au journalisme canadien. Comme le précise Gottlieb,

[i]n South African context, embedded translation is found [...] in Afrikaans newspapers [...].

[...] embedded translation includes instances where parts of the translated passages retain their original form and protrude from the text like barren peaks, nunataks, in a glacial landscape. (Gottlieb 2010: 202)

Pour des raisons d'uniformité, nous n’avons pas retenu le terme nunatak pour notre terminologie.

Nous entendons par paraphrase interlinguistique le développement explicatif en langue cible d'un discours prononcé en langue source. L'exemple suivant est lui aussi tiré de l'entrevue de Pierre Elliott Trudeau en 1970, citée dans La Presse.

(18) M. Trudeau a reconnu qu'il se trouve beaucoup de cœurs tendres qui n'aiment pas particulièrement la présence de gens armés.

(Desjardins 1970: F1, voir annexe)

La précision en langue source correspond à une explication en langue source qui vient éclairer le sens d'une expression traduite vers une langue cible donnée. L'exemple suivant porte encore sur l'entrevue accordée par Pierre Elliott Trudeau en 1970, dans Le Devoir cette fois.

(19) Trudeau - C’est vrai qu'il y a beaucoup de cœurs tendres («bleeding hearts») dans la place, qui ne peuvent supporter la vue de personnes qui portent des casques et des fusils.

(Le Devoir 1970: 11, voir annexe; nous soulignons)

Quant à la citation bilingue, il s'agit d'une citation directe, retranscrite dans un article à la fois en langue source et en langue cible. L'extrait suivant, tiré d'un article de la Gazette, contient un exemple de citation bilingue. Le journaliste Graham Fraser y faisait le compte rendu du discours de la défaite de René Lévesque, lors du référendum sur la souveraineté en 1980.

(20) The vote had hardly changed all night - but at 8:45 with the No vote holding at 58 per cent, the crowd began the old Independantiste chant of "Ce n'est qu'un debut, continuons le combat." (This is only the beginning, let's continue the struggle.)

(Fraser 1980: 1, voir annexe; nous soulignons)

La plupart des exemples présentés ci-dessus portent sur l'entrevue donnée par Pierre Elliott Trudeau en 1970. C'est que cet événement fit couler beaucoup d'encre. Le journal Le Devoir écrivit à ce sujet:

(21) Au moment où il allait faire son entrée à la Chambre des communes mardi, M. Pierre Elliott Trudeau, fut invité, de façon impromptue, à commenter la 
présence aux abords du parlement de nombreux membres des forces armées canadiennes. Au cours de la conversation qui suivit, le Premier ministre du Canada a fait des déclarations très importantes sur la crise soulevée par l'enlèvement de MM. Cross et Laporte. Voici le texte intégral de l'interview accordée par M. Trudeau à MM. Tim Ralphe du réseau anglais de Radio-Canada, et Peter Reilly, de la station CJOH-TV d'Ottawa, dans une version française établie par Le Devoir.

(Le Devoir 1970: 11, voir annexe)

Rares sont les entrevues qui sont ainsi transcrites intégralement dans les pages d'un journal. Encore plus rares sont les occasions où un journal établit lui-même la traduction d'un texte politique canadien. Il faut dire que cette entrevue a produit une vive impression au Canada et qu'elle a été rediffusée dans tous les médias canadiens. Encore aujourd'hui, des extraits en sont cités (surtout en anglais, mais aussi en français) pour évoquer l'attitude du Premier ministre relativement à la Crise d'octobre.

Dans la traduction française de cette entrevue de 1970, dans Le Devoir (exemple [19]), la langue du texte original est utilisée pour préciser le sens de la traduction française. Au Canada, on ne recourt qu'occasionnellement à la langue source pour expliciter une expression traduite. Ce type d'intervention est possible parce qu'une partie non négligeable du lectorat montréalais peut lire l'anglais. D’ailleurs, cet exemple est unique en son genre, comme on peut le voir dans le tableau 1 ci-dessous. Ce tableau présente les différents types d'intervention traductionnelle dans le souscorpus. Lorsqu'un type d'intervention apparait au moins une fois dans un article, la case correspondante du tableau est marquée d'un X.

TABleau 1

Types d'intervention traductionnelle dans le corpus

\begin{tabular}{|c|c|c|c|c|c|}
\hline & Traduction & $\begin{array}{c}\text { Paraphrase } \\
\text { interlinguistique }\end{array}$ & $\begin{array}{c}\text { Précision en } \\
\text { langue source }\end{array}$ & $\begin{array}{l}\text { Citation } \\
\text { bilingue }\end{array}$ & $\begin{array}{c}\text { Non- } \\
\text { traduction }\end{array}$ \\
\hline $\begin{array}{r}\text { La Presse } \\
\text { Desjardins (1970) } \\
\text { La Presse (1990) }\end{array}$ & $\mathrm{X}$ & $\mathrm{X}$ & & & $\frac{\mathrm{X}}{\mathrm{X}}$ \\
\hline $\begin{array}{l}\text { Le Devoir } \\
\qquad \begin{array}{l}\text { Le Devoir }(1970) \\
\text { Le Devoir }(1990)\end{array}\end{array}$ & $\frac{X}{X}$ & & $\mathrm{X}$ & & \\
\hline $\begin{array}{l}\text { The Globe and Mail } \\
\text { Cleroux (1980) } \\
\text { The Globe and Mail (1990) } \\
\text { The Globe and Mail (1995c) } \\
\text { Picard (1995b) }\end{array}$ & \begin{tabular}{r}
$\mathrm{X}$ \\
\hdashline $\mathrm{X}$ \\
\hdashline $\mathrm{X}$ \\
\end{tabular} & $\begin{array}{r}X \\
\hdashline \\
\hdashline-1 \\
\hdashline\end{array}$ & & & \\
\hline $\begin{array}{r}\text { The Gazette } \\
\text { Fraser (1980) } \\
\text { Authier (1990) } \\
\text { Norris et Block (1995) } \\
\text { Authier et Scott (1995) }\end{array}$ & $\frac{X}{X}$ & 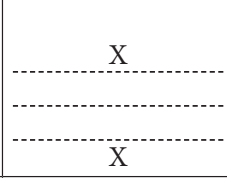 & & 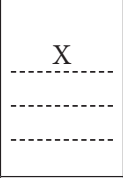 & X \\
\hline $\begin{array}{l}\text { Total } \\
12 \text { articles }\end{array}$ & 9 & 5 & 1 & 1 & 3 \\
\hline
\end{tabular}

Dix ans après la Crise d'octobre, le journaliste Graham Fraser a dû rendre compte d'un discours non traduit. Le journaliste évoque alors le discours de la défaite de René Lévesque dans The Gazette: 
(22) a. "We have to swallow it this time, it's not easy," he said. "It is clear that the people of Quebec want to give the federal system another chance."

b. But he told the audience not to give up the goal of sovereignty.

c. With a final call of "A la prochaine" - until next time - he left the stage [...].

(Fraser 1980: 1, voir annexe)

Graham Fraser a effectué trois types d'interventions traductionnelles dans son article, soit la traduction (a), la paraphrase interlinguistique (b) et la citation bilingue (c). Même si le journaliste ne précise pas qu'il a lui-même traduit les extraits du discours, on le devine facilement à la façon dont il dispose ses citations bilingues. En effet, cet agencement d'interventions montre au lectorat que le discours a été prononcé en français et que les premières citations étaient en fait des traductions. Notons qu'à l'instar de la précision en langue source de l'exemple (19), la citation bilingue (“"A la prochaine” - until next time») est fort rare (voir le tableau 1). De plus, dans les deux types d'intervention, le journaliste présume que le lectorat ne sera pas importuné par la présence d'une langue étrangère dans l'article.

Contrairement à son confrère de la Gazette, le journaliste Richard Cleroux du Globe and Mail cite ce discours de René Lévesque en anglais seulement. Par ailleurs, dans le même article, il cite aussi Claude Ryan et Pierre Elliott Trudeau.

(23) Mr. Levesque was almost weeping when he told an emotional rally at Montreal's Paul Sauve arena last night: "This hurts more than any election defeat. I know of what I speak." [...]

Mr. Levesque said last night the Quebec people had "clearly given federalism another chance" and called upon Prime Minister Pierre Trudeau to make good of his promises of constitutional reform. [...]

Liberal Leader Claude Ryan, the leader of the no forces supporting federalism, told a flag-waving victory rally at the Verdun Auditorium that the outcome showed "the depth of our Canadian roots," and that Quebeckers believe that "our future lies within federalism." [...]

Mr. Trudeau [...] called on Quebeckers to heal their wounds: "To all my fellow Quebeckers who have been wounded by defeat, I wish to say that we have all lost a little in this referendum. [...]"

(Cleroux 1980: 1, voir annexe)

Le texte de Richard Cleroux a l'avantage de l'homogénéité: le lecteur n'est pas gêné par la présence d'une langue étrangère, ce qui facilite la lecture. Ses interventions sont de l'ordre de la traduction et de la paraphrase interlinguistique. Rappelons que le lectorat du Globe and Mail connaît moins bien le français que celui de la Gazette. L'article du Globe donne cependant l'impression que tous les locuteurs se sont exprimés en anglais. Or, seuls Ryan et Pierre Elliott Trudeau ont exprimé une partie de leur discours dans la langue de Shakespeare. Le lecteur ne peut donc pas faire la différence entre les traductions officielles et les traductions du journaliste. Pourtant, en tant que représentant d'un gouvernement provincial unilingue, René Lévesque a fait le choix politique au soir du référendum de 1980 de ne s'exprimer qu'en français. À certains égards, la présentation homogène de monsieur Cleroux entraîne une distorsion des événements.

En 1990, le Premier ministre du Québec, Robert Bourassa, convoque une conférence de presse afin de s'adresser aux Québécois, à la suite de l'échec de l'Accord du lac Meech. Le Premier ministre s'était principalement adressé en français à la population, mais il avait également prononcé quelques paroles en anglais. Dans les archives 
électroniques de l'Assemblée nationale du Québec, on retrouve une "copie révisée» de cette conférence de presse. Aucune traduction n'apparaît dans ce document. Au lendemain du discours, tant The Globe and Mail que Le Devoir retranscrivent dans leurs pages le discours de Robert Bourassa. Voici les intertitres utilisés par ces deux quotidiens en guise d'introduction du discours:

(24) From an address given on television Saturday by Quebec Premier Robert Bourassa:

(The Globe and Mail 1990: A13, voir annexe)

(25) Voici le texte intégral de la déclaration du Premier ministre Robert Bourassa faite samedi après-midi au Salon rouge de l'Assemblée nationale en réaction à l'échec de l'accord du lac Meech.

(Le Devoir 1990: 13, voir annexe)

Dans The Globe, le discours est seulement retranscrit en anglais, alors que dans Le Devoir, il ne l'est qu'en français. Puisque le gouvernement ne semble pas avoir fourni de traduction de ce discours ${ }^{10}$, il faut conclure que les deux quotidiens ont eu recours à la traduction: un paragraphe pour Le Devoir et la majorité du discours pour The Globe and Mail. On notera ici que le discours du Globe ne correspondait pas aux normes de lisibilité et d'idiotisme exigées par la traduction professionnelle. Le texte anglais présente certaines tournures maladroites, ou très proches du français ${ }^{11}$ :

(26) [...] nous posions cinq conditions, ni plus, ni moins, sans aucune arrière pensée de marchandage. Comme le disait fort opportunément hier le chef de l'Opposition, le gouvernement du Québec et son chef se trouvaient à prendre un risque avec l'histoire.

[...] we presented five conditions, no more, no less, with no intention to bargain. As the leader of the opposition said opportunely yesterday, the government of Quebec and its leader found themselves taking a risk with history.

(The Globe and Mail 1990: A13)

L'expression «to bargain» ne correspond pas à l'usage conventionnel en anglais. On dira davantage "no intention of bargaining». Par ailleurs, «opportunely» et «taking a risk» sont trop calqués sur le français. Pour nous conformer aux règles d'écriture de l'anglais, nous proposons «aptly said» et «taking a chance». Comme le souligne Brian Mossop (1990), il existe de nombreuses traductions journalistiques de mauvaise qualité. Mossop attribue en partie ce problème au fait que les journalistes n'ont pas de formation en traduction. Il pense aussi que les journaux accordent moins d'importance à la qualité de la langue de l'analyse qu'auparavant. Il ajoute qu'un texte mal traduit peu porter atteinte à la crédibilité du message.

Les deux autres quotidiens ont choisi un type différent d'intervention, soit la non-traduction plutôt que la traduction. Pour préciser la langue d'allocution du discours, le journaliste de la Gazette a écrit:

(27) Bourassa, who opened his news conference with a "Mes chers compatriotes," was given a thunderous ovation by about 80 MNAs when he and his wife, Andrée, arrived.

(Authier 1990: A1, voir annexe)

Dans le reste de son article, Philip Authier utilise les propos tenus en anglais par le Premier ministre lors d'une conférence de presse. De cette façon, il n'a pas eu à traduire le discours unilingue de Robert Bourassa. Quant au quotidien La Presse, il 
a publié le texte de Robert Bourassa tel que prononcé, avec des parties en français et en anglais.

En 1995, le discours français de Lucien Bouchard fut traduit vers l'anglais par The Globe and Mail (voir l'exemple [15] ci-dessus), même si le chef de l'Opposition avait également prononcé un discours d'expression anglaise. Traditionnellement, lorsque le Premier ministre du Canada prononce un message à la nation et que le chef de l'Opposition y répond, les discours sont télévisés et présentés simultanément dans les deux langues officielles. Cette simultanéité symbolise à bien des égards l'idée que les deux versions du discours sont identiques, qu'il s'agit du même discours. Or, en traduisant vers l'anglais une partie du discours français de Lucien Bouchard, le quotidien torontois fait voir à son lectorat que l'homme politique québécois avait livré deux messages différents. D'ailleurs, The Globe and Mail publie également des extraits du discours en anglais:

(28) The following is a partial of the speech Lucien Bouchard delivered in English.

(The Globe and Mail 1995c: A16, voir annexe)

Puisque que les extraits (15) et (28) figurent sur la même page (A16) dans le quotidien, tout est mis en place pour faciliter la comparaison. Par ailleurs, sur cette même page, figure également le discours de Jean Chrétien en anglais:

(29) The Prime Minister delivered this speech in English and in French.

(The Globe and Mail 1995b: 16, voir annexe)

En agençant les discours de la sorte, The Globe and Mail fait comprendre à son lectorat que le chef de l'Opposition n'a pas respecté la norme qui veut qu'un seul message bilingue soit envoyé à tous les Canadiens.

Quelques jours après le discours de Lucien Bouchard, le 30 octobre 1995, les journalistes d'expression anglaise ont tous traduit les propos du Premier ministre Jacques Parizeau, qui concédait (maladroitement) la victoire du parti du NON au référendum. Voici trois extraits représentatifs, deux tirés de The Gazette et une autre du Globe and Mail:

(30) In his address to rowdy Yes-side supporters after conceding at the Palais des Congrès, Parizeau declared: "We are beaten, it's true. But by what? By money and the ethnic vote."

(Norris et Block 1995: A1, voir annexe)

(31) Wiping away tears and standing before 3,000 stunned sovereignists the way René Lévesque did 15 years ago, Parizeau said the Yes side came within an inch of winning and the battle will continue.

(Authier et Scott 1995: B1, voir annexe)

(32) "It's true that we were beaten, but by whom? Money and the ethnic votes," he told Yes supporters. He spoke ominously of the "temptation for revenge" and promised to "exact revenge" for the loss by never abandoning the dream to build a francophone nation in Quebec.

(Picard 1995b: A1, voir annexe)

Dans l'extrait (30), on retrouve un exemple de traduction, tandis que dans l'extrait (31), il s'agit d'une paraphrase interlinguistique. L'extrait (32) contient les deux types d'intervention. Contrairement à ce qui s'est passé lors du référendum de 1980, les textes sont tous homogènes d'un point de vue traductologique. Dans les articles 
consultés, les journalistes ne précisent pas la langue dans laquelle s'est exprimé Parizeau, ni le fait qu'ils ont eux-mêmes traduit les propos du Premier ministre.

\subsection{Conclusion provisoire}

Dans un article publié en 1990, Brian Mossop explique que les journalistes n'ont pas les mêmes objectifs que les traducteurs. Il cite en exemple une lettre du Premier ministre René Lévesque, traduite par The Globe and Mail. Dans sa traduction vers l'anglais, le quotidien a consciemment ou non présenté à son lectorat une voix étrangère, c'est-à-dire québécoise française, au Canada anglais. En d'autres termes, la traduction du Globe était trop calquée sur le français pour être idiomatique, ce qui est contraire à la doctrine des traducteurs professionnels. Cette démarche a entre autres eu pour effet politique de mettre le lectorat en présence du caractère «autre» du texte.

En fait, on pourrait ajouter que les journalistes et les traducteurs professionnels effectuent des quêtes de sens différentes. Pour les journalistes, le sens va au-delà du texte: il faut livrer l'information dans toute sa complexité, dans toute sa dimension culturelle et politique. C'est ce qui amène un journaliste à multiplier les interventions traductionnelles, en fonction de la réalité à décrire. Pour le traducteur professionnel, le sens réside seulement dans le texte à transmettre, en fonction des normes des cultures sources et cibles.

Par ailleurs, plusieurs interventions traductionnelles de notre corpus appuient les affirmations de Brian Mossop. En effet, la non-traduction, la citation bilingue et la précision en langue source contribuent toutes à rendre explicite le phénomène de contact des langues au Canada. Même si ce n'est pas intentionnel, la traduction non idiomatique participe également à ce phénomène. L'effet politique de ces interventions est appréciable: grâce à elles, le lecteur sait, du moins jusqu'à un certain point, qu'il est en présence d'une réalité culturelle et linguistique différente de la sienne. Il faut cependant souligner que les occurrences de traduction idiomatique et de paraphrase interlinguistique sont légion dans le corpus. C'est donc qu'il y a une homogénéisation non négligeable du discours des hommes politiques québécois. En taisant les différences de l'autre communauté linguistique officielle, on entretient le phénomène des deux solitudes, où chaque communauté linguistique s'épanouit dans son univers, sans se soucier de l'autre. Dans un contexte bilingue comme le Canada, ce genre de situation contribue à bien des égards à séparer les communautés et peut engendrer des problèmes de communication. Ceci étant dit, en minimisant les contacts entre les communautés, on évite les confrontations et on rassure les gens en accordant un statut important à leur langue maternelle. La compilation des résultats au Tableau 1 semble démontrer que les quotidiens Le Devoir et The Globe and Mail participent davantage à l'homogénéisation du discours que La Presse ou The Gazette: les premiers font un usage quasi exclusif de la traduction et la paraphrase interlinguistique, alors que les seconds utilisent en plus la non-traduction et la citation bilingue. Vu le corpus restreint de notre recherche, cette observation doit toutefois être prise en contexte, voire réexaminée à la lumière d'un corpus plus étendu.

\section{Conclusion}

Alors, d'un point de vue traductologique, quel(s) rôle(s) jouent les journalistes lorsqu'ils sont en présence de discours politiques canadiens? Les résultats obtenus 
jusqu'ici (dans Gagnon 2012 et dans le présent article) suggèrent trois rôles: la mise en lumière de l'activité de traduction, le commentaire sur la traduction et les interventions traductionnelles. En d'autres termes, le journaliste coiffe tour à tour le chapeau de l'éclairagiste, du commentateur et du traducteur. Dans notre corpus, ces rôles sont parfois assumés individuellement, parfois simultanément.

Lorsque le journaliste tient le rôle du traducteur, c'est son point de vue de journaliste (ou celui de son journal) qui est exprimé et non celui d'une institution gouvernementale. On a vu qu'à l'occasion, le traducteur-journaliste fournit à son lectorat des outils d'analyse (précision en langue source, non-traduction, citation bilingue) pour mieux comprendre les relations entre les hommes politiques et les communautés linguistiques auxquelles ces derniers s'adressent. Soulignons aussi un fait important: dans notre corpus, ce sont les discours d'hommes politiques québécois que les journalistes traduisent. Certaines traductions journalistiques donnent l'occasion de mettre en valeur la spécificité québécoise, alors que d'autres masquent ces attributs. Sous la plume d'un journaliste, il arrive aussi que la traduction tienne lieu de commentaire. C'est le cas de l'exemple (15), où la traduction d'un texte de Lucien Bouchard vers l'anglais correspond à une critique de sa traduction officielle. En d'autres termes, les journalistes du Globe and Mail ont jugé défavorablement les divergences entre les versions linguistiques de son discours. Notons par ailleurs que les cas réels de traductions commentées sont rares en journalisme. En effet, même lorsque les journalistes endossent le rôle de commentateur, ils parviennent difficilement à dissiper l'illusion que le narrateur de la traduction n'appartient pas à la même culture que son lectorat.

Dans la section 2, nous avions émis l'hypothèse que le gouvernement fédéral publiait les discours du Premier ministre dans des documents séparés afin de décourager les comparaisons entre les versions française et anglaise des discours. Vu le petit nombre de comparaisons effectuées par les journalistes de la presse écrite (6 articles sur un total de 106), on peut en déduire que cette stratégie, s'il s'agit en effet d'une stratégie, a généralement bien fonctionné. En outre, dans ses communiqués, le gouvernement fédéral présente tous les discours du Premier ministre comme des originaux et les journalistes ne remettent presque jamais cette pratique en question. Seuls les Comptes rendus des débats mentionnent la traduction, mais ils ne sont généralement pas préparés à l'intention des journalistes comme le sont les communiqués. On pourrait brièvement étudier la soumission tacite des journalistes à l'aide du modèle d'analyse d'Herman et de Chomsky (1998: 2), qui vise à comprendre les liens existants entre le pouvoir, la richesse et les médias. Ce modèle porte sur la propagande: «[it] traces the routes by which money and power are able to filter out the news fit to print, marginalize dissent, and allow government and dominant private interests to get their messages across to the public». En outre, Herman et Chomsky ont identifié les cinq filtres qui contribuent à définir ce qui mérite d'être signalé dans les nouvelles. L'un de ces filtres porte entre autres sur la confiance accordée par les journalistes aux institutions gouvernementales: "the reliance of the media on information provided by government, business, and "experts" funded and approved by these primary sources and agents of power» (Herman et Chomsky 1998: 2). En somme, on pourrait affirmer que parce que les traductions proviennent du gouvernement fédéral, on leur accorde une très grande confiance. C'est peut-être ce qui explique la raison pour laquelle certains journalistes affirment que les versions française et anglaise d'un discours sont 
similaires alors que ce n'est pas nécessairement le cas: la confiance qu'accordent les journalistes aux traductions fédérales porte peut-être atteinte à leur sens critique?

Les résultats obtenus dans la présente étude semblent démontrer que les journalistes canadiens ont tout de même contribué à comprendre le phénomène de la traduction des discours politiques au $\mathrm{xx}^{\mathrm{e}}$ siècle. Or il semble que ces contributions ne soient pas seulement une chose du passé, comme le montre l'extrait suivant, tiré d'un article du Devoir paru en 2008:

(33) M. Harper a conclu son adresse en s'attaquant à la coalition comprenant le Bloc québécois. «Une coalition avec les souverainistes ne peut pas aider le Canada. Et les partis de l'opposition n'ont pas le droit démocratique d'imposer une coalition avec les souverainistes.» En anglais, il a utilisé le mot «séparatistes», seule différence entre les deux textes.

(Buzzetti 2008: A10, voir annexe)

Le discours du Premier ministre Stephen Harper a été prononcé en pleine crise parlementaire, en décembre 2008. Stephen Harper savait que l'expression anglaise "separatists» avait une connotation très négative, tant au Canada anglais qu'au Canada français. Pendant cette crise, le gouvernement du Canada a entre autres remis en cause la légitimité du Bloc québécois, un parti souverainiste élu au gouvernement fédéral par un nombre important de Québécois. En fait, l'expression «separatists» s'inscrivait dans un discours de nature francophobe tenu au Canada anglais tout au long de la crise (voir aussi Wheeldon 2011 : 69-70). Dans ce contexte, le commentaire de la journaliste souligne non seulement l'écart de traduction entre les deux versions du discours, mais aussi l'écart d'idéologie.

En outre, les journalistes du Xxi ${ }^{\mathrm{e}}$ siècle semblent encore s'adonner à la traduction, comme le démontrent les exemples suivants. Il s'agit de la traduction d'un discours du chef démissionnaire du Bloc Québécois, Gilles Duceppe. Ce discours a été prononcé en français le 2 mai 2011 à l'issue des élections fédérales du Canada.

(34) "I'm leaving, but others will follow, until Quebec becomes a country," Mr. Duceppe told party faithful in a short speech at a sombre election night rally in Montreal on Monday night.

(Leblanc 2011: A9; nous soulignons, voir annexe)

(35) As a result, Gilles Duceppe immediately announced his resignation as leader of the sovereigntist party, declaring in his concession speech: "I am leaving, but others will follow, until Quebec becomes a party."

(Blaze et Boswell 2011: A1; nous soulignons, voir annexe)

Le premier exemple (34) est tiré du Globe and Mail, alors que le second (35) provient d'un quotidien canadien-anglais apparu au début du second millénaire, le National Post. Dans les deux quotidiens, les journalistes ont traduit le discours de Duceppe vers l'anglais, sans préciser que l'homme politique ne s'est exprimé qu'en français. Or, l'article du National Post contient un non-sens (until Quebec becomes a party). Sans contexte, cette erreur en page couverture donne une image négative de l'ancien chef de parti: on a l'impression que Duceppe s'est mal exprimé en anglais ou que sa pensée était incohérente. Le lecteur ne sait pas qu'il s'agit d'une faute des auteurs de l'article. Puisque le National Post s'affiche ouvertement contre le mouvement souverainiste, cette maladresse, même involontaire, contribue au discours antisouverainiste du journal. 
En somme, les dimensions politiques, culturelles et sociales du rôle des journalistes en traduction ne sont pas à négliger: d'un coup de crayon, les journalistes ont la possibilité de contribuer au rapprochement ou à l'éloignement des communautés linguistiques du Canada. Aujourd'hui, avec le Web, les journalistes ont aisément accès aux versions anglaise et française d'un discours. Il est possible que cette nouvelle accessibilité facilite l'étude de la traduction des discours et augmente le nombre de commentaires des journalistes à ce propos. Nous souhaitons donc vivement d'autres études de ce genre pour obtenir un tableau d'ensemble plus conséquent.

\section{NOTES}

1. Marissal, Vincent (16 novembre 2002): Le français, langue seconde. La Presse. 119(28):B2.

2. Canada, Prime minister (1970a): Notes for a National Broadcast by the Prime Minister Pierre Elliott Trudeau, Friday, 16 October, 1970. Communiqué. Ottawa: Office of the Prime Minister, 1-8.

3. Canada, Premier ministre (1970b): Notes pour un message du Premier ministre Pierre Elliott Trudeau à la nation, vendredi, le 16 octobre 1970. Communiqué. Ottawa: Cabinet du Premier ministre, 1-10.

4. Canada, Prime minister (1980a): Statement by the Prime Minister Pierre Elliott Trudeau in Response to the Quebec Referendum Vote, May 20, 1980. Communiqué. Ottawa: Office of the Prime minister, 1-3.

5. Canada, Premier ministre (1980b): Déclaration du Premier ministre Pierre Elliott Trudeau le soir du référendum québécois, le 20 mai 1980. Communiqué. Ottawa: Cabinet du Premier ministre, 1-3.

6. Canada, Prime minister (1990a): Notes for an Address to the nation by Prime Minister Brian Mulroney, Ottawa, June 23, 1990. Communiqué. Ottawa: Office of the Prime Minister, 1-3.

7. Canada, Premier ministre (1990b): Notes pour une adresse à la nation du premier minister Brian Mulroney, Ottawa, le 23 juin 1990. Communiqué. Ottawa: Cabinet du Premier ministre, 1-3.

8. Canada, Prime minister (1995a): Address to the nation by Prime Minister Jean Chrétien, October 25, 199. Communiqué. Ottawa: Office of the Prime Minister, 1-4.

9. Canada, Premier ministre (1995b): Message à la nation du Premier ministre Jean Chrétien, le 25 octobre 1995. Communiqué. Ottawa: Cabinet du Premier ministre, 1-4.

10. Archives politiques du Québec (Dernière mise à jour : 2012): Consulté le 12 juin 2013, <http://www. archivespolitiquesduquebec.com/discours/p-m-du-quebec/robert-bourassa/conferences-depresse-1989-1994/>.

11. Nous tenons à remercier Monsieur Brendan Brogan, traducteur indépendant vers l'anglais, pour son avis professionnel sur la question.

\section{RÉFÉRENCES}

Bernier, Marc-François (2004): Éthique et déontologie du journalisme. Édition revue et augmentée. Québec: Presses de l’Université Laval.

Conway, Kyle (2011) : Everyone Says No: Public Service Broadcasting and the Failure of Translation. Montréal: McGill-Queen's University Press.

DAvies, Nick (2008) : Flat Earth News. Londres: Chatto \& Windus.

FAirclough, Norman (1995) : Media Discourse. Londres: Edward Arnold.

GaGnon, Chantal (2006a): Ideologies in the history of translation: a case study on Canadian political speeches. In: Paul Bandia et Georges BAstin, dir. Charting the Future of Translation History. Ottawa: University of Ottawa Press, 201-223.

Gagnon, Chantal (2006b): Language plurality as power struggle, or: Translating politics in Canada. Target. International Journal of Translation Studies. 18(1):69-90.

Gagnon, Chantal (2009): La traduction des discours politiques au Canada. Thèse de doctorat non publiée. Birmingham: Aston University.

Gagnon, Chantal (2012): La visibilité de la traduction au Canada en journalisme politique: mythe ou réalité? Meta. 57(4):943-959.

Gottlieb, Henrik (2010): Multilingual Translation vs. English-fits-all in South African Media. Across Languages and Cultures. 11(2):189-216. 
Herman, Edwards S. et Chomsky, Noam (1998): Manufacturing Consent, the Political Economy of the Mass Media. New York: Pantheon Books.

Herman, Thierry (2009): Le discours politique est-il soluble dans le discours médiatique? Les communiqués de presse des partis gouvernementaux helvétiques. In: Marcel Burger, Jérôme JACQUin, Raphaël MiCHELI, éd. Les médias et le politique. (Colloque Le français parlé dans les médias: les médias et le politique, 1-4 septembre 2009). Lausanne: Centre de linguistique et des sciences du langage. Consulté le 24 juin 2011, <http://www.unil.ch/clsl/ page81503.html>.

Jacobs, Geert (1999): Preformulating the News. Philadelphie: John Benjamins Publishing Company.

Mossop, Brian (1990): Translating institutions and “idiomatic" translation. Meta. 35(2):342-355.

Pander MaAt, Henk (2007): How Promotional Language in Press Releases is Dealt With by Journalists. Genre Mixing or Genre Conflict? Journal of Business Communication. 44(1):5995.

Potvin, Maryse (1999): Les dérapages racistes à l'égard du Québec au Canada anglais depuis 1995. Politique et sociétés. 18(2):101-132.

Robinson, Gertrude J. (1998): Constructing the Quebec referendum: French and English Media Voices. Toronto: University of Toronto Press.

Van Dijk, Teun A. (1988): News as Discourse. Hillsdale, NJ, Erlbaum.

Van Hout, Tom, Pander Maat, Henk et De Preter, Wim (2011): Writing from news sources: The case of Apple TV. Journal of Pragmatics. 43:1876-1889.

WheELdone, Johannes (2011): Actors, targets, and guardians: Using routine activities theory to explore the 2008 decision to prorogue parliament in Canada. Canadian Journal of Sociology/ Cahiers canadiens de sociologie. 36(1):59-93.

\section{Annexe: corpus d'articles de journaux cités}

\section{2}

Canadian Press (8 avril 1942): 'Fight now to defend existence,' Mr. King Requests 'Free Hand' to Guide Canada's War Plans. The Globe and Mail. 99(28 802):1-2.

Canadian Press (10 avril 1942): Cardin wants Quebec to lead in voting 'yes'. The Globe and Mail. 99(28 804):1-2.

Canadian Press (25 avril 1942a): Cardin Nips Conscription Bogey In Urging Quebec to Vote 'Yes'. The Gazette. 171(99):1;23.

Canadian Press (25 avril 1942b): Warns Quebec Reign of King Rests on Vote. The Globe and Mail. 99(28 817):1.

The GaZette (10 avril 1942): Cardin advises French Canada lead 'YES' Vote. 171(86):1;19.

\section{0}

Canadian Press (17 octobre 1970): Text of PM's address to the nation. The Gazette. 193:8.

Desjardins, Marcel (14 octobre 1970): La société doit prendre tous les moyens pour se défendre contre l'émergence d'un pouvoir parallèle. La Presse. 86(238):F1

Goldblatt, Murray et Burns, John (17 octobre 1970): PM defends proclamation. The Globe and Mail. 127(37 708):1-2.

LE DEVOIR (15 octobre 1970): Seuls les faibles reculeront devant la mise en veilleuse de certaines libertés civiles (Trudeau). 61(239):11.

Presse Canadienne (17 octobre 1970): La déclaration de M. Trudeau. Le Devoir. 61(241):6.

Turcotte, Claude (17 octobre 1970): Trudeau: céder, ce serait encourager le terrorisme. La Presse. 86(241):A1-A2.

\section{6}

BissonnetTe, Lise (25 novembre 1976) : La vraie question est ailleurs... Le partage des pouvoirs ne suffira pas à arrêter le PQ (Trudeau). Le Devoir. 68(275):1;6. 
Canadian Press (25 novembre 1976): Text of Trudeau's speech. The Gazette. 199:9.

Desjardins, Lionel (16 novembre 1976): Trudeau prêt à collaborer. La Presse. 93(20):1-3.

1977

Cousineau, Louise (25 janvier 1977): Pour Lévesque, on sabre dans le hockey. La Presse. 93(20):A6.

1980

Cleroux, Richard (21 mai 1980): Federalists get close to 60 per cent. The Globe and Mail. 137(40 702):1-2.

Cousineau, Louise (1980): Après tant de passions déchaînées, une soirée dénuée d'émotion. La Presse. 96(120):11.

Fraser, Graham (21 mai 1980): It's not easy to swallow: Levesque. The Gazette. 202:1.

Turcotte, Claude (21 mai 1980) : Il faut rebâtir la maison sans plus tarder (Trudeau). Le Devoir. 71(114):1;14.

1990

Authier, Philip (24 juin 1990): Bourassa slams door on constitution talks. The Gazette. A1;A7. LA PRESSE (24 juin 1990): Déclaration de Bourassa. 106(241):B2.

LE DeVoIr (24 juin 1990): Désormais, des négociations à deux. Mon seul guide sera l'intérieur supérieur du Québec. 81(146):13.

The Globe and MaIL (25 juin 1990) : A critical moment in Quebec's history. 147(43 854):A13.

1995

Authier, Philip et Scott, Sarah (31 octobre 1995): Parizeau: Most francophones votes Yes. The Gazette. B1;B7.

Dion, Jean (31 octobre 1995): Chrétien: au Canada de ne pas laisser tomber les Québécois. Le Devoir. A1;A10.

HÉBERT, Chantal (26 octobre 1995): Ultime appel. La Presse. 112(7):A1-A2.

Norris, Alexander et Block, Irwin (31 octobre 1995): Premier's remarks shock minorities. The Gazette. A1;A4.

PICARD, André (26 octobre 1995a): PM pleads with undecided voters. The Globe and Mail. $152: A 1 ; A 8$.

PiCARD, André (31 octobre 1995b) : NO - by a whisker. Parizeau promises to 'exact revenge' for sovereingtist loss. The Globe and Mail. 152:A1-A2.

The Globe and Mail (26 octobre 1995a): Prime Minister can't be trusted, Bouchard says. 152:A16.

THE GLOBE AND MAIL (26 octobre 1995b): Why destroy Canada? 152:A16.

The Globe and MAIL (26 octobre1995c): Wind of panic blowing in Ottawa, Bouchard says. 152:A16

Wells, Paul (26 octobre 1995): Canada the best: PM. But Bouchard sees two solitudes, The Gazette. A1;A11.

\section{8}

Buzzetti, Hélène (4 décembre 2008): La volonté des électeurs doit être respectée. Le Devoir. 99(277):A10.

\section{1}

Blaze Carlson, Kathryn et Boswell, Randy (3 mai 2011): Quebec takes the plunge with NDP; Duceppe quits. The National Post. 13(156):A1.

Leblanc, Daniel (3 mai 2011): Duceppe resigns as Bloc leader after losing riding. The Globe and Mail. A9. 